\title{
Spatial Memory in Captive Giant Anteaters (Myrmecophaga tridactyla)
}

\author{
Stephanie M. Allard ${ }^{1,2 *}$ Julie L. Earles ${ }^{2}$, and Leilani DesFosses ${ }^{3}$ \\ ${ }^{1}$ Detroit Zoological Society \\ ${ }^{2}$ Florida Atlantic University \\ ${ }^{3}$ University of South Florida, Tampa \\ *Corresponding author (Email: sallard@dzs.org ) \\ Citation - Allard, S. M., Earles, J. L., \& DesFosses, L. (2014). Spatial memory in captive giant anteaters \\ (Myrmecophaga tridactyla) Animal Behavior and Cognition, 1(3), 331-340. doi: 10.12966/abc.08.08.2014
}

\begin{abstract}
The type of learning exhibited during foraging has been studied in a large number of species. Species that feed on food sources that temporally vary in quality could be well suited for exhibiting evidence of spatial learning. The foraging strategies of captive giant anteaters were examined using an experimental foraging task. Two giant anteaters were exposed to a modified radial arm maze in order to determine whether or not they would demonstrate evidence of spatial learning. Both subjects demonstrated significant improvement in performance by visiting baited feeders more consistently across learning trials. A disruption in performance occurred when the task was reversed, indicating that giant anteaters may use spatial learning to locate food sources. Obtaining a more sound understanding of the cognitive abilities of giant anteaters may help to enhance their welfare in captive settings.
\end{abstract}

Keywords - Giant anteater, Spatial memory, Radial arm maze, Cognition, Welfare

To be successful in the wild, animals must overcome various challenges that impact their survival. Locating sufficient food is one challenge that evolutionarily drives cognitive skills and behavior. The summation of environmental factors and physiological needs shapes an animal's foraging strategies to achieve a balance between energy expenditure and gained nutrients from acquired food. These factors include, but are not limited to, territory size, competition, type of food consumed, and metabolism of the animal (Pyke, 1984). Giant anteaters (Myrmecophaga tridactyla) are specialized carnivores, feeding exclusively on ants and termites (Redford, 1985). They will feed for short periods of time from numerous different locations, which is necessitated by the defense mechanisms of their prey. However, the means by which giant anteaters locate their food are currently unknown. They could use one or a combination of olfactory, visual, or spatial cues to find enough food to meet their daily energy demands. Understanding how giant anteaters forage could therefore provide clues to the cognitive abilities of giant anteaters.

One early study attempted to discern the acuity of the sense of smell of giant anteaters. Researchers used a T-maze and two scents, camphor and eucalyptus, to indicate positive and negative cues that would lead to a food reward or nothing. During the experiment, researchers blended the two scents and varied the concentrations to determine when the giant anteater could no longer discriminate between the two scents. These results were then compared to similar discrimination tests in human subjects. The researchers concluded that giant anteaters likely have a sharper sense of smell than humans, but they were unable to further specify their olfactory capabilities or the extent to which giant anteaters rely on this strategy (McAdam \& Way, 1967). Based on these results, olfactory cues could be instrumental in the process of locating food. 
Depending on the exact acuity of their senses of sight and smell, using visual cues while foraging could be a faster and more energy efficient strategy of locating food than relying on olfactory cues. Many species from birds and rats to numerous non-human primate species have been found to use spatial memory in addition to or instead of other environmental cues to locate food sources (birds, Bednekoff, Balda, Kamil, \& Hile, 1997; rats, Olton \& Samuelson, 1976; gibbons, Asensio, Brockelman, Malaivijitnond, \& Reichard, 2011; yellow-nosed monkeys, MacDonald \& Wilkie, 1990; capuchins, Janson, 1998; gorillas, Gibeault \& MacDonald, 2000). With such widespread use of spatial memory, it is plausible that giant anteaters could also be utilizing spatial memory when foraging.

Giant anteater territorial patterns could be indicative of spatial memory in this species. If giant anteaters feed from a more or less fixed number of food sources in a confined area, they would likely have some method of remembering where ant and termite hills are located. Field studies that have used VHF radio-telemetry and GPS to monitor activity patterns and habitat use in giant anteaters have revealed that they utilize specific home ranges (Medri \& Mourao, 2005; Shaw, Machado-Neto, \& Carter, 1987). Because they remain within specific home ranges, giant anteaters could have spatial cognitive capabilities used for remembering the location of known food sources and for remembering which sources they have recently depleted. Although giant anteaters have clear home ranges, the territories of neighboring individuals can overlap (Mourao \& Medri, 2007; Shaw, Carter, \& Machado-Neto, 1985). Insufficient research has been conducted to determine if giant anteaters must compete with each other for food resources, but researchers observed an encounter between two giant anteaters in what they hypothesized was a territory defense display (Shaw et al., 1985). Competition for food sources would also provide evolutionary drive to develop spatial memory to improve foraging efficiency.

Additionally, giant anteaters mark their territories by scratching trees, which could be a form of communication between giant anteaters with overlapping territories (Braga, Santos, \& Batista, 2010). In addition to the defensive display witnessed by Shaw and colleagues (1985), these markings could be a method for giant anteaters to claim particular areas. This behavior could, however, also be used for communication for mating purposes. Although a correlation between prey sources and markings has not yet been found, scratching trees could also be a visual signal for locating food. With multiple potential motivations behind marking and little research on the subject, determining with certainty whether this behavior contributes to territory and resource defense is difficult. Marking could nonetheless be linked to visual foraging cues or to spatial mapping.

Food preference could also influence the foraging strategies utilized by giant anteaters. Both wild and captive giant anteaters exhibited food preference between different species of ants and termites, but these preferences did not match in the two settings. Their food preference in the wild depended on nutritional value, availability, and response to attack (Redford, 1985). Consequently, giant anteaters could have strategies for remembering the locations of mounds that house favored species of ants and termites, particularly if they experience competition in overlapping territories. Rats are capable of remembering the location of preferred foods (Bird, Roberts, Abroms, Kit, Crupi, 2003). Free-ranging mountain gorillas not only show spatial memory skills, but also display preference for foraging in areas that contain higher quality food (Vedder, 1984). Spatial memory would be similarly beneficial for giant anteaters to locate preferred food sites.

As the primary food of giant anteaters, ant and termite hills act as a self-replenishing food source. Previous research indicates that animals that feed on similar self-replenishing food sources appear to have some dependence on spatial cognitive abilities. The hummingbird, for example, must rely on spatial memory and replenishment rates to know at which flowers they will find food (Sutherland \& Gass, 1995). Additional studies on species of nectar-feeding bats produced similar results regarding spatial memory and replenishment rates even in the presence of other cues (Thiele \& Winter, 2005). Fruit is also a selfreplenishing food source, and mangabeys have been found to utilize spatial memory when searching for fruiting trees (Janmaat, Byrne, \& Zuberbuhler, 2006a). The mangabeys preferentially selected trees that were not recently depleted, suggesting that they rely on memory not only to locate trees, but to keep track of food source quality as well. Rates of refruiting are impacted by temperature, and these primates will revisit previously depleted trees more rapidly following warmer weather (Janmaat, Byrne, \& Zuberuhler, 
2006b). Anteaters visit numerous insect nests each day, and the level of damage caused to the nests is generally low enough to allow the insects to make repairs within a few hours (Naples, 1999). Because their food sources are self-replenishing, anteaters could be employing similar strategies when foraging for ants and termites to know which nests would have sufficiently recovered from a previous feeding.

By providing a foraging task in a zoo setting, this study aimed to determine if giant anteaters are capable of using spatial memory to locate past food sources. With this knowledge, field biologists could have a better understanding of the feeding habits and the travel patterns of wild giant anteaters.

\section{Method}

\section{Subjects}

The subjects of this study were two captive adult male giant anteaters, henceforth known as Male A and Male B. Both giant anteaters were captive born and mother-raised. Typically, the giant anteaters were fed a gruel mixture to substitute for their natural diet, along with various enrichment items, such as avocado. During the course of the study, the avocado was not used with the diet so that it could be used for the experiment. The remaining diet was fed to the anteaters following their regular routine.

Both males were singly housed in separate exhibits without direct contact with other animals. Male A's enclosure was adjacent to an otter exhibit, but the otters were never present during testing. Male B's enclosure was off-exhibit, but shared a barrier with another exhibit that contained tapirs and various species of waterfowl. The neighboring animals never appeared to distract Male B from the experiment. Male A's enclosure included an open area where testing was conducted, a pool, a small cave, and various trees and logs with controlled access to his night house. Male B's enclosure consisted mostly of open grassy area with trees on the far end from the night house.

\section{Apparatus}

The testing apparatus was made of PVC pipe with an opaque plastic lid attached by a hinge. A bungee cord attached to the lid created tension so that the lid would close as soon as the animal was not actively holding the feeder open. The tension of the bungee cord was carefully monitored to make sure the lid did not snap back with force and hurt the animals. Sixteen feeders were used in this experiment, eight per subject. To ensure that there was no visual difference between the feeders, all feeders used white supplies and green bungee cords. Each apparatus was approximately $10.15 \mathrm{~cm}$ in diameter with a 15.25 $\mathrm{cm}$ diameter lid. The feeders rested inside a $15.25 \mathrm{~cm}$ diameter PVC pipe buried into the ground in order to prevent the giant anteaters from moving the feeders. Although the feeders were removed at the end of each trial, the buried pipes remained in the ground. Caps were places on the buried pipes to prevent the giant anteaters from stepping in the holes and injuring themselves. Before each trial, a tablespoon of avocado was placed into each of the baited feeders. This totaled approximately half an avocado per trial. Avocado was selected to bait the feeders because it is a preferred treat of the giant anteaters in this study. The subjects were therefore highly motivated to complete foraging tasks. At the end of each trial, the feeders were repaired if necessary and washed with soap and water to eliminate any odor.

\section{Test Area}

A modified radial arm maze (Lipp et al., 2001; Olson \& Samuelson, 1976; Perdue, Snyder, Zhihe, Marr, \& Maple, 2011) was used in which the feeders were arranged in a circular grid pattern with a diameter of approximately $10 \mathrm{ft}$. The test area in Male A's enclosure was located in an open dirt area at one end of the exhibit nearest to the door of his night house. The feeders were arranged around a wooden post with a tree and logs as additional landmarks. The test area in Male B's enclosure was located in the middle of the enclosure in a grassy area with trees on one side and his night house on the other side. The feeders were similarly arranged around a tree with logs on one side to serve as a landmark. 


\section{Data Collection}

The experiment was conducted seven days a week from approximately 8:00 am to 10:00 am. A single observer (L. DesFosses) with no previous experience working with giant anteaters collected data each day. In order to keep temporal factors as consistent as possible, data for Male A was always collected first, followed immediately by data collection for Male B. Continuous focal sampling using paper, a pen, and a stopwatch was used to collect data during the experiment. The main behavior of interest was visiting the feeder, which required a paw or muzzle under the lid of a feeder. Any unusual behavior or interruptions to a trial were also noted. A trial began as soon as the subject entered the testing area and ended once all four baited feeders were successfully visited.

In the spatial learning phase of the experiment, every other feeder was baited, for a total of four baited feeders. The same four feeders were baited each day until the giant anteaters were able to successfully choose three baited feeders out of their first four feeder interactions for four out of five consecutive days.

The second phase of the study was a reversal phase conducted to see if the anteaters use spatial memory for successful foraging. The four originally baited feeders were left empty, while the originally unbaited feeders were baited. This phase of the study continued until the subjects correctly identified three baited feeders in their first four selections for three out of four consecutive days.

When baiting the feeders, the experimenter walked around the perimeter of the circular array with the avocado to ensure there was no scent trail leading specifically to baited feeders. The feeder that the experimenter baited first varied each day. During the trials, the experimenter stood outside the exhibit in a location that was far enough away so as not to distract the giant anteaters from the experiment.

\section{Statistical Analyses}

Graphic analysis was used to evaluate the learning of each anteater. We used a montecarlo simulation with 1,000,000 trials to determine the random foraging rate of selecting the baited feeders in the first four feeder interactions. The random foraging rate was determined to be 1.65 out of 4 baited feeders selected in the first four feeder interactions. For both learning and reversal, two-tailed one sample $t$-tests were calculated to compare the mean number of baited feeder interactions in the subjects' first four interactions in the first five trials and the mean number of baited feeder interactions in the last five trials to the expected value due to random foraging (i.e., 1.65). The alpha level was set at 0.05 for all analyses.

\section{Results}

As can be seen in Figure 1, Male A took 23 trials to meet the learning criterion of at least three out of four baited feeder interactions in the first four feeder interactions he made for four out of five consecutive days. It is clear that his performance improved across learning trials, decreased dramatically for the first reversal trial, and recovered by the $12^{\text {th }}$ reversal trial.

As can be seen in Figure 2, Male B took 27 trials to meet the learning criterion of at least 3 out of 4 baited feeder interactions in the first four feeder interactions for four other of five consecutive days. His performance also decreased when the baited feeders were reversed, and it took him until the $18^{\text {th }}$ reversal trial to recover.

\section{Learning Trials}

For Male A, the average number of visits to baited feeders in the first four feeder interactions in the first 5 trials $(M=1.20, S D=0.84)$ was not significantly different from the expected number based on random foraging (i.e., 1.65), $t(4)=1.20, p=0.295$. The average number of baited feeder interactions in the first four interactions in the last 5 trials $(M=2.80, S D=0.45)$ was significantly higher than the expected number based on random foraging (i.e., 1.65), $t(4)=5.75, p=0.005$. As can be seen in Figure 1, 
the number of visits to baited feeders in the first four interactions in the last 5 trials was higher than the number of visits to baited feeders in the first 5 trials.

For Male B, the average number of visits to baited feeders in the first four feeder interactions in the first 5 trials $(M=1.80, S D=0.45)$ was not significantly different from the expected number based on random foraging (i.e., 1.65), $t(4)<1.0$. The average number of baited feeder interactions in the first four interactions in the last 5 trials $(M=2.80, S D=0.45)$ was significantly higher than the expected number based on random foraging (i.e., 1.65), $t(4)=5.75, p=0.005$. As can be seen in Figure 2, the number of visits to baited feeders in the first four interactions in the last 5 trials was significantly higher than the number of visits to baited feeders in the first 5 trials.

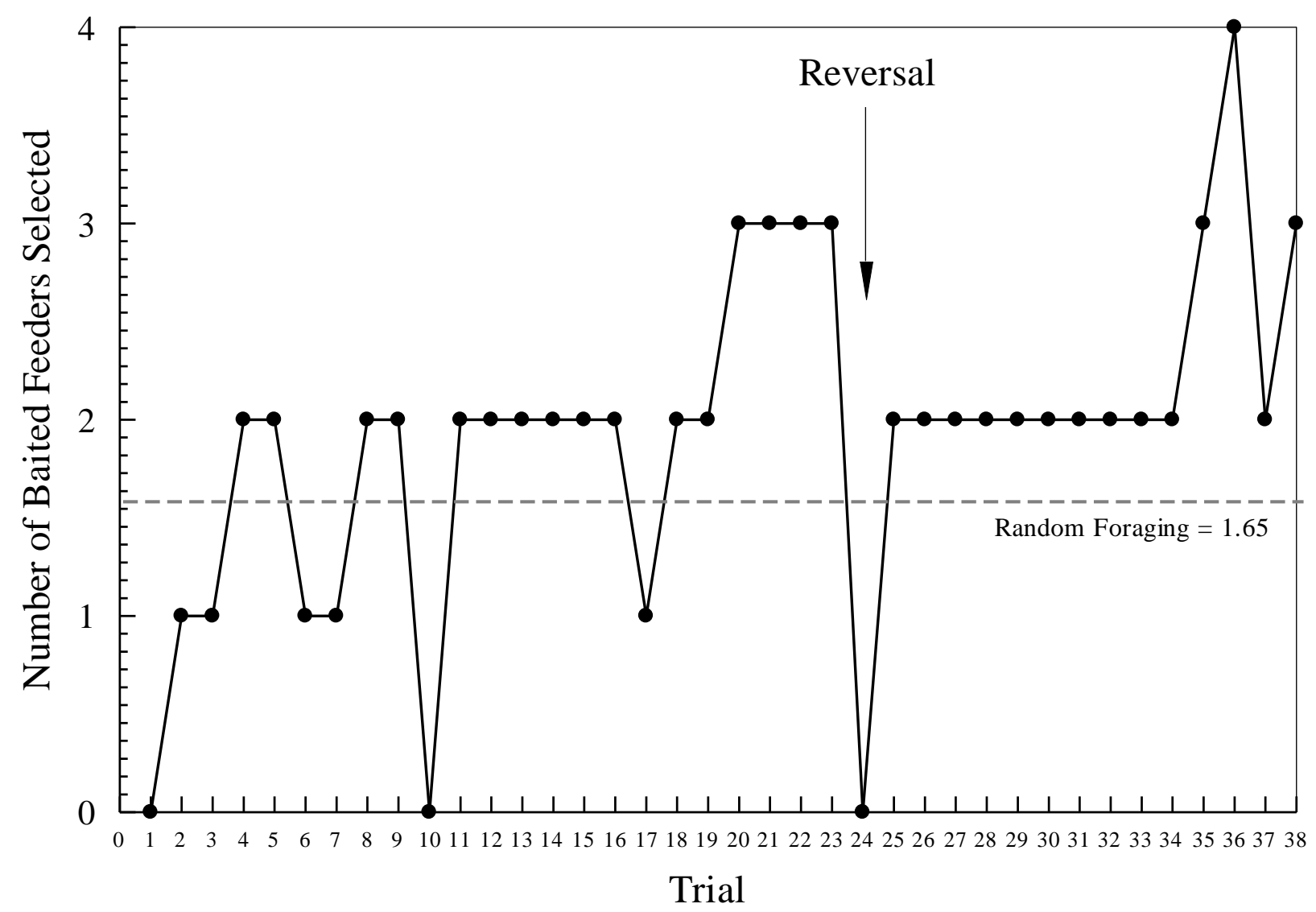

Figure 1.Male A success in first four selections

\section{Reversal Trials}

For Male A, the average number of visits to baited feeders in the first four feeder interactions in the first 5 trials of the reversal phase $(M=1.60, S D=0.89)$ was not significantly different from the expected number based on random foraging (i.e., 1.65), $t(4)<1.0$. The average number of baited feeder interactions in the first four interactions in the last 5 trials of the reversal phase $(M=2.80, S D=0.84)$ was significantly higher than the expected number based on random foraging (i.e., 1.65), $t(4)=3.07, p=.037$. As can be seen in Figure 1, for Male A, the number of visits to baited feeders in the first four interactions in the last 5 trials was significantly higher than the number of visits to baited feeders in the first 5 trials.

Male B, made 2 visits to baited feeders in the first four feeder interactions in all of the first 5 trials of the reversal phase. Due to this lack of variability, a t-test could not be used to compare his performance to random foraging. The average number of baited feeder interactions in the first four interactions in the 
last five trials of the reversal phase $(M=2.60, S D=0.55)$ was significantly higher than the expected number based on random foraging (i.e., 1.65), $t(4)=3.88, p=0.018$. As can be seen in Figure 2, for Male $\mathrm{B}$, the number of visits to baited feeders in the first four interactions in the last 5 trials was higher than the number of visits to baited feeders in the first 5 trials.

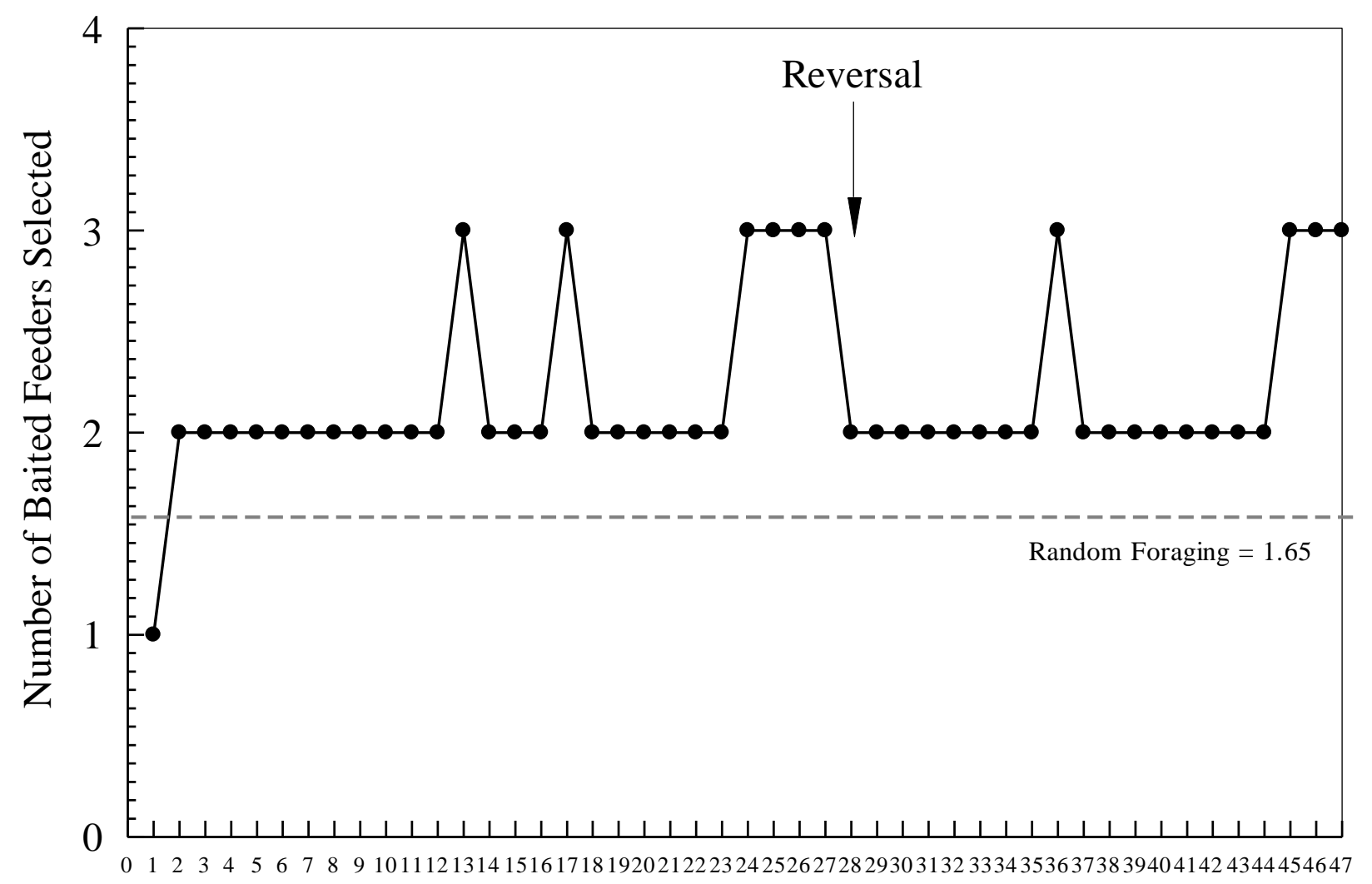

Trial

Figure 2. Male B success in first four selections

\section{Discussion}

The results suggest that spatial memory does, in fact, play a role in the foraging behavior of giant anteaters. For both the learning phase and the reversal phase, both Male A and Male B visited fewer baited feeders in the first five than in the last five trials of the phase. Thus both anteaters showed improved spatial memory for the baited feeders across trials.

During the reversal phase, Male A immediately traveled to the feeders that were no longer baited and opened them. Male B also opened unbaited feeders in this phase of the study. The giant anteaters' inspection of previously baited feeders when the locations were reversed suggests that they were not simply relying on smell to forage for food. The behavior in this reversal phase suggests that giant anteaters can also use spatial memory for returning to known food sources.

There were individual differences between Male A and Male B in regard to their foraging techniques. Male A appeared to require more time to master manipulating the feeders, while Male B manipulated the feeders skillfully and opened them with ease from the first day. Despite Male B's dexterity with the feeders, Male A reached the efficiency targets faster. Male B seemed to have a more inquisitive personality and explored more of the unbaited feeders even when he achieved his target. This 
characteristic could have contributed to Male B's apparent slower learning. Because Male B is younger than Male A, age also could have affected performance.

Although both subjects required over 20 days to reach criteria, they did seem to learn before they reached efficiency. Both giant anteaters experienced environmental disturbances on days landscaping or loud noises occurred near their exhibits. On these days, the giant anteaters would travel straight to the baited feeders. Their performance on these days was higher regardless of their point in the experiment (e.g., day 36 on Figure 1; days 13, 17, and 36 on Figure 2). Therefore, the giant anteaters could have learned which feeders had food earlier then when they reached criteria, but continued to check additional feeders anyway. In a zoo setting, the anteaters have no time restrictions or limitations on their food consumption. Consequently, they may lack the motivation to find food quickly and efficiently (see also Zamisch \& Vonk, 2012).

Because this study was limited to two subjects, additional research with more subjects, including female giant anteaters, should be conducted to verify the findings reported here. Giant anteaters born in different settings could perform differently on a foraging task like the one in this study. Giant anteaters born in the wild might retain more of their instincts to quickly locate and consume food, and thus reach efficiency faster than anteaters born in captivity. Conversely, giant anteaters that were raised by humans instead of their mothers could perform similarly or even slower than the captive born giant anteaters in this study. Further research could examine the differences among wild-born, captive-born, and handraised giant anteaters. Additionally, this study could be expanded to include visual markers on the feeders to determine if the giant anteaters could use those cues to locate food. Further research could also explore the importance of olfactory cues in foraging strategies of giant anteaters.

This type of research also has the potential to improve the welfare of captive giant anteaters, as better captive management practices can ensue based on a clearer understanding of the giant anteaters' cognitive abilities. Despite advances over the last several decades, captive environments can still be structurally simple and often unresponsive to behaviors of the animals inhabiting them. Such environments do not allow animals to interact with their surroundings in ways that promote the development and use of sensory and cognitive abilities (Morgan \& Tromborg, 2008). This can result in compromised abilities to exercise choice and control over the environment.

There continues to be growing evidence that providing choice and control can provide substantial behavioral benefits to captive animals (Bassett \& Buchanan-Smith, 2007; Buchanan-Smith \& Badihi, 2012; Meehan \& Mench, 2007; Ross, 2006; Spinka \& Wemelsfelder, 2011; Watters, 2009), resulting in enhanced welfare. Choice and control can be increased for captive animals by using appropriate challenges for the animals (Meehan \& Mench, 2007), and food acquisition is one area that can provide such challenges in captivity (Bashaw, Bloomsmith, \& Maple, 2003; Watters, Miller, \& Sullivan, 2011).

Wild animals can spend significant portions of their time foraging and actively consuming food items. Wild giant anteaters must not only locate insect nests, but they must also safely obtain the insects from their nests. This requires the animal to use physical and cognitive skills. In captivity, food is often provided in predictable ways and in forms that are vastly different than they would be in the wild (Kagan \& Veasey, 2010). Captive diets typically require very little effort to obtain and consume and therefore offer little challenge for the animals. In captivity, giant anteaters are typically fed a nutritionally complete diet, balanced for their specific insectivore requirements, and often presented as gruel. Foraging and consumption can therefore be very different for captive anteaters than for their wild counterparts. If giant anteaters do, in fact, rely on cognitive abilities to locate food sources, presenting them with foraging opportunities that allow them to use those abilities could benefit their welfare. Overcoming these types of relevant challenges can be intrinsically rewarding for captive animals (Langbein, Siebert, \& Nurnberg, 2009; Manrod, Hartdegen, \& Burghardt, 2008).

The body of research into the interaction of welfare and cognition is growing (e.g., Bethell, Holmes, Maclarnon, \& Semple, 2012; Broom, 2010; Clark, 2011; Herrelko, Vick, \& Buchanan-Smith, 2012; McEwen \& Sapolsky, 1995; Mendl, 1999; Yamanashi \& Hayashi, 2011), but more attention still needs to be placed on whether captive animals are being sufficiently cognitively challenged. As stated by Ross, "working to improve captive animal care without an understanding of how animal minds work, of 
the scope in which they perceive and interact with their social and physical environment is akin to drawing a map without knowledge of landmarks and bearings" (2010, p. 310). Studies designed to measure cognitive abilities have the potential to not only help to determine the cognitive abilities of the animals, but also to provide direct enrichment benefits to the animals. Devices used in such experiments provide animals with activities that can decrease boredom and increase exploration, factors that have been linked to enhanced wellbeing (Celli, Tomonoga, Udono, Teramoto, \& Nagano, 2003).

Enrichment specifically designed to stimulate cognitive abilities has been shown to improve welfare in a variety of species (e.g., dolphins, Clark, Davies, Midigan, Warner, \& Kuczaj, 2013; great apes, Gilloux, Gurnell, \& Shepherdson, 1992; piglets, Zebunke, Puppe, \& Langbein, 2013). Cognitive challenges have the most beneficial effects on welfare if the level of difficulty is appropriate for an animal's skill level (Clark, 2011). Determining the cognitive capabilities of both species and individual animals is therefore critical if we are to present animals with relevant and appropriately difficult challenges in captivity. Although integrating problem solving opportunities into the lives of captive animals can be challenging, a more comprehensive understanding of their cognitive abilities can render this more feasible.

\section{Conclusion}

This study provides evidence that giant anteaters could rely on spatial memory in order to return to previously visited food sources. These findings are in keeping with previous literature involving other species (e.g., Janmaat et al., 2006a; Sutherland \& Gass, 1995; Thiele \& Winter, 2005; Tarou, 2003) and show promise for future research on the spatial memory of giant anteaters. The results of this study also have the potential to enhance the welfare of captive giant anteaters by providing more insight into their cognitive abilities, information that can subsequently be used to create new opportunities for environmental and cognitive stimulation.

\section{References}

Asensio, N., Brockelman, W. Y., Malaivijitnond, S., \& Reichard, U. H. (2011). Gibbon travel paths are goal oriented. Animal Cognition, 14, 395-405.

Bashaw, M. J., Bloomsmith, M. A., \& Maple, T. L. (2003). To hunt or not to hunt? A feeding enrichment experiment with captive large felids. Zoo Biology, 22, 189-198.

Bassett, L., \& Buchanan-Smith, H. M. (2007). Effects of predictability on the welfare of captive animals. Applied Animal Behaviour Science, 102, 223-245.

Bednekoff, P. A., Balda, R. P., Kamil, A. C., \& Hile, A. G. (1997). Long-term spatial memory in four seed-caching corvid species. Animal Behaviour, 53, 335-341.

Bethell, E., Holmes, A., Maclarnon, A., \& Semple, S. (2012). Cognitive bias in a non-human primate: Husbandry procedures influence cognitive indicators of psychological well-being in captive rhesus macaques. Animal Welfare, 21, 185-195

Bird, L. R., Roberts, W. A., Abroms, B., Kit, K. A., \& Crupi, C. (2003). Spatial memory for food hidden by rats (Rattus norvegicus) on the radial maze: Studies of memory for where, what, and when. Journal of Comparative Psychology, 117, 176-187.

Braga, F. G., Santos, R. E. F., \& Batista, A. C. (2010). Marking behavior of the giant anteater Myrmecophaga tridactyla (Mammalia: Myrmecophagidae) in Southern Brazil. Zoologia, 27, 7-12.

Broom, D. M. (2010). Cognitive ability and awareness in domestic animals and decisions about obligations to animals. Applied Animal Behaviour Science, 126, 1-11.

Buchanan-Smith, H. M., \& Badihi, I. (2012). The psychology of control: Effects of control over supplementary light on welfare of marmosets. Applied Animal Behaviour Science, 137, 166-74.

Celli, M. L., Tomonoga, M., Udono, T., Teramoto, M., \& Nagano, K. (2003). Tool use task as environmental enrichment for captive chimpanzees. Applied Animal Behaviour Science, 81, 171-182.

Clark, F. E. (2011). Great ape cognition and captive care: Can cognitive challenges enhance well-being? Applied Animal Behaviour Science, 135, 1-12. 
Clark, F. E., Davies, S. L., Midigan, A. W., Warner, A. J., \& Kuczaj, S. A. (2013). Cognitive enrichment for bottlenose dolphins (Tursiops truncatus): Evaluation of a novel underwater maze device. Zoo Biology, 32, 608-619.

Gibeault, S., \& MacDonald, S. E. (2000). Spatial memory and foraging competition in captive Western lowland gorillas (Gorilla gorilla gorilla). Primates, 41, 147-160.

Gilloux, I., Gurnell, J., \& Shepherdson, D. (1992). An enrichment device for great apes. Animal Welfare, 1, 279289.

Herrelko, E. S., Vick, S.-J., \& Buchanan-Smith, H. M. (2012). Cognitive research in zoo-housed chimpanzees: Influence of personality and impact on welfare. American Journal of Primatology, 74, 828-840

Janmaat, K. R. L., Byrne, R. W., \& Zuberbuhler, K. (2006a). Evidence for spatial memory of fruiting states of rainforest trees in wild mangabeys. Animal Behaviour, 72, 797-807.

Janmaat, K. R. L., Byrne, R. W., \& Zuberbuhler, K. (2006b). Primates take weather into account when searching for fruits. Current Biology, 16, 1232-1237.

Janson, C. H. (1998). Experimental evidence for spatial memory in foraging wild capuchin monkeys, Cebus appela. Animal Behavior, 55, 1229-1243.

Kagan, R., \& Veasey, J. (2010). Challenges of zoo animal welfare. In D. G. Kleiman, K. V. Thompson, \& C. Kirk Baer (Eds.), Wild mammals in captivity: Principles and techniques for zoo management (p.11-21). Chicago: The University of Chicago Press.

Langbein, J., Siebert, K., \& Nurnberg, G. (2009). On the use of an automated learning device by group-housed dwarf goats: Do goats seek cognitive challenges? Applied Animal Behaviour Science, 120, 150-158.

Lipp, H., Pleskacheva, M. G., Gosswiler, H., Ricceri, L., Smirnova, A. A., Garin, N. N., ... Kuptsov, P. A. (2001). A large outdoor radial maze for comparative studies in birds and mammals. Neuroscience and Biobehavioral Reviews, 25, 83-99.

Manrod, J. D., Hartdegen, R., \& Burghardt, G. M. (2007). Rapid solving of a problem apparatus by juvenile blackthroated monitor lizards (Varanus albigularis albigularis). Animal Cognition, 11, $267-273$.

MacDonald, S. E., \& Wilkie, D. M. (1990). Yellow-nosed monkeys, (Cercopithecus ascanius whitesidei) spatial memory in a simulated foraging environment. Journal of Comparative Psychology, 104, 382-387.

McAdam, D. W., \& Way, J. S. (1967). Olfactory discrimination in the giant anteater. Nature, 12, 316-317.

McEwen, B. S., \& Sapolsky, R. M. (1995). Stress and cognitive function. Current Opinion in Neurobiology, 5, 205216.

Medri, I. M., \& Mourao, G. (2005). Home range of giant anteaters (Myrmecophaga tridactyla) in the Pantanal wetland, Brazil. Journal of Zoology, 266, 365-373.

Meehan, C. L., \& Mench, J. A. (2007). The challenge of challenge: Can problem solving opportunities enhance animal welfare? Applied Animal Behaviour Science, 102, 246-261.

Mendl, M. (1999). Performing under pressure: Stress and cognitive function. Applied Animal Behaviour Science, 65, 221-244.

Morgan, K. N., \& Tromborg, C. T. (2008). Sources of stress in captivity. Applied Animal Behaviour Science, 102, 262-302.

Mourao, G., \& Medri, I. M. (2007). Activity of a specialized insectivorous mammal (Myrmecophaga tridactyla) in the Pantanal of Brazil. Journal of Zoology, 271, 187-192.

Naples, V. L. (1999). Morphology, evolution and function of feeding in the giant anteater (Myrmecophaga tridactyla). Journal of Zoology, 249, 19-41.

Olton, D. S., \& Samuelson, R. J. (1976). Remembrance of places passed: Spatial memory in rats. Journal of Experimental Psychology: Animal Behavior Processes, 2, 97-116.

Perdue, B. M., Snyder, R. J., Zhihe, Z., Marr, M. J., \& Maple, T. L. (2011). Sex differences in spatial ability: A test of the range size hypothesis in the order Carnivora. Biology Letters, 7, 380-383.

Pyke, G. H. (1984). Optimal foraging theory: A critical review. Annual Review of Ecology and Systematics, 15, 523575.

Redford, K. H. (1985). Feeding and food preference in captive and wild giant anteaters (Myrmecophaga trydactyla). Journal of Zoology, 205, 559-572.

Ross, S. R. (2006). Issues of choice and control in the behaviour of a pair of captive polar bears (Ursus maritimus). Behavioural Processes, 73, 117-120.

Ross, S. R. (2010). How cognitive studies help shape our obligation for ethical care of chimpanzees. In E. V. Londsorf, S. R. Ross, \& T. Matsuzawa (Eds.), The mind of the chimpanzees: Ecological and experimental perspectives (pp. 309-319). Chicago: The University of Chicago Press. 
Shaw, J. H., Carter, T. S., \& Machado-Neto, J. C. (1985). Ecology of the giant anteater Myrmecophaga tridactyla in Serra da Canastra, Minas Gerais, Brazil: A pilot study. In G. G. Montgomery (Ed.), The evolution and ecology of armadillos, sloths and vermilinguans. Washington: Smithsonian Institution Press.

Shaw, J. H., Machado-Neto, J. C., \& Carter, T. S. (1987). Behavior of free-living giant anteaters (Myrmecophaga tridactyla). Biotropica, 19, 255-259.

Spinka, M., \& Wemelsfelder, F. (2011). Environmental challenge and animal agency. In M. C. Appleby, J. A. Mench, I. A. S. Olsson, \& B. O. Hughes (Eds.), Animal welfare, $2^{\text {nd }} e d$ (pp. 27-43). Cambridge, UK: CABI Publishing,.

Sutherland, G. D., \& Gass, C. L. (1995). Learning and remembering of spatial patterns by hummingbirds. Animal Behavior, 50, 1273-1286.

Thiele, J., \& Winter, Y. (2005). Hierarchical strategy for relocating food targets in flower bats: Spatial memory versus cue-directed search. Animal Behavior, 69, 315-327.

Tarou, L. R. (2003). An examination of the role of associative learning and spatial memory in foraging in two species of bear (family: Ursidae) (unpublished doctoral dissertation). Georgia Institute of Technology, Atlanta, GA.

Vedder, A. L. (1984). Movement patterns of a group of free-ranging mountain gorillas (Gorilla gorilla beringei) and their relation to food availability. American Journal of Primatology, 7, 73-88.

Watters, J. V. (2009). Toward a predictive theory for environmental enrichment. Zoo Biology, 28, 609-622.

Watters, J. V., Miller, J. T., \& Sullivan, T. J. (2011). Note on optimizing environmental enrichment: A study of fennec fox and zoo guests. Zoo Biology, 30, 647-654.

Yamanashi, Y. \& Hayashi, M. (2011). Assessing the effects of cognitive experiments on the welfare of captive chimpanzees (Pan troglodytes) by direct comparison of activity budget between wild and captive chimpanzees. American Journal of Primatology, 73, 1231-1238.

Zamisch, V., \& Vonk, J. (2012). Spatial memory in captive American black bears (Ursus Americanus). Journal of Comparative Psychology, 126, 372-387.

Zebunke, M., Puppe, B., \& Langbein, J. (2013). Effects of cognitive enrichment on behavioural and physiological reactions of pigs. Physiology \& Behavior, 118, 70-79. 